\title{
SOME AXIALLY SYMMETRIC STRESS DISTRIBUTIONS IN ELASTIC SOLIDS CONTAINING PENNY-SHAPED CRACKS
}

\author{
III. A CRACK IN A CIRCULAR BEAM
}

by $\mathrm{W}$. D. COLLINS

(Received 14th November 1961)

\section{Introduction}

This paper concludes the investigation of axially symmetric stress distributions in elastic solids containing penny-shaped cracks, commenced in previous papers (1), (2), by considering the stress distribution in a circular beam containing a crack opened by internal pressure or by uniform tension. The method of analysis, developed in the previous papers, is to first seek a representation of the displacement at a point of the beam as a sum of two terms, one of which is a representation of the displacement due to the crack in an otherwise unbounded infinite solid whilst the second is a general representation of the displacement in an undamaged beam, and then to show that this representation satisfies the conditions on the crack and the curved surface of the beam provided an unknown function occurring in it is the solution of a certain Fredholm integral equation. This equation holds whatever the ratio of the radius of the crack to that of the beam, but is most readily solved by iteration when this ratio is small, this solution being a perturbation on that for a crack in an infinite solid.

The required representation of the displacement is given in $\$ 2$ and the governing Fredholm equation of the problem obtained in $\S 3$. In $\S 4$ we suppose the crack opened by a constant internal pressure and give expressions for the normal displacement over the surface of the crack and for the increase in the potential energy of the beam when the radius of the crack is small compared with that of the beam. Finally, in $\$ \mathbf{5}$ we consider the crack in a beam under uniform tension and determine the critical value of the tension at which the crack becomes unstable by means of the Griffith criterion, the ratio of the radii of the crack and the beam again being small.

\section{The Representation of the Displacement}

We use cylindrical polar coordinates $(\varpi, \theta, z)$ with the centre and axis of the crack as origin and $z$-axis, so that the crack, radius $a$, is given by

$$
z=0(0 \leqq \varpi \leqq a)
$$


and the curved surface of the beam, radius $c(c>a)$, by $m=c(-\infty<z<\infty)$, and adopt Love's notation (3, p. 79) for the stress components at a point of the beam. Thus, for example, the components of the stress vector across an element whose normal is in the $z$-direction are $\hat{\boldsymbol{m} z}$ and $\hat{z z}$, the component in the $\theta$-direction being zero since the stress distribution is torsion-free. At all interior points of the beam the stresses and the components $u$ and $w$ of the displacement $\boldsymbol{D}$ in the $\varpi$ - and $z$-directions satisfy the body stress equations and the stress-strain relations for an isotropic material, the displacement components being $0\left(|z|^{-1}\right)$ at a large distance $|z|$ from the origin. Further, the displacement and stress components are continuously differentiable at all interior points of the beam and are continuous for approach to points on either face of the crack, except that on its edge $(z=0, m=a)$ the stresses can be expected to tend to infinity as $\left(a^{2}-\varpi^{2}\right)^{-\frac{1}{2}}$. We suppose the crack is opened by an axisymmetric normal pressure, so that

$$
\hat{\varpi z}=0, \quad \hat{z z}=-p(\varpi), \quad \text { on } z=0(0 \leqq \varpi \leqq a),
$$

$p(\varpi)$ being a given continuous function of $w$, whilst the curved surface of the beam is stress-free, giving

$$
\hat{\varpi} \tilde{\omega}=\hat{\varpi z}=0 \text { on } \varpi=c(-\infty<z<\infty) .
$$

We construct the displacement $D$ at any point of the beam as

$$
D=D_{1}+D_{2},
$$

where $D_{1}$ is a representation of the displacement at a point in an infinite solid containing a crack opened under normal pressure and $D_{2}$ is a general representation of the displacement at a point in the undamaged beam $\varpi=c \quad(-\infty<z<\infty)$, the $z$-component of $D_{2}$ being antisymmetric about $z=0$ because of (2.1). The representation $D_{1}$ has already been found as (1, equation (2.3))

$$
D_{1}=(3-4 \eta) \phi k-z \operatorname{grad} \phi+\operatorname{grad} \psi,
$$

where $\boldsymbol{k}$ is the unit vector in the $z$-direction, $\eta$ is Poisson's ratio, and $\phi(w, z)$ and $\psi(\varpi, z)$ are harmonic functions, given by

$$
\begin{aligned}
\frac{\partial \psi}{\partial z} & =-(1-2 \eta) \phi, \\
\phi(\varpi, z) & =\frac{1}{2 i} \int_{-a}^{a} \frac{g(t) d t}{\left(\varpi^{2}+(z+i t)^{2}\right)^{\frac{1}{2}}}, \\
\psi(\varpi, z) & =-\frac{(1-2 \eta)}{2 i} \int_{-a}^{a} g(t) \log \left[\left(\varpi^{2}+(z+i t)^{2}\right)^{\frac{1}{2}}+z+i t\right] d t .
\end{aligned}
$$

Here $g(t)$ is a real continuous odd function as yet undetermined, the square roots have non-negative real parts (1, equations (2.9) and (2.10)), and the logarithm its principal value. 
We now require a general representation of the displacement at a point in an undamaged beam. Previous workers on elastostatic problems for circular beams (4), (5), have usually represented the displacement in terms of a biharmonic stress function (3, pp. 274-6), but it seems preferable to use a representation deduced by Barton (6) from the Papkovich-Neuber solution of the equations of elasticity. We have as a representation for $D_{2}$

$$
\boldsymbol{D}_{2}=(3-4 \eta) \tau \boldsymbol{m}-\boldsymbol{m} \operatorname{grad} \tau+\operatorname{grad} \chi,
$$

where $\boldsymbol{w}$ is the unit vector in the $w$-direction, and $\chi(\varpi, z)$ and $\tau(\varpi, z) \cos \theta$ are harmonic functions, that is, $\tau(\varpi, z)$ is a solution of the equation

$$
\left(\frac{\partial^{2}}{\partial \varpi^{2}}+\frac{1}{w} \frac{\partial}{\partial \varpi}+\frac{\partial^{2}}{\partial z^{2}}-\frac{1}{w^{2}}\right) \tau=0
$$

We represent $\chi$ and $\tau$ as

$$
\begin{aligned}
& \chi(\varpi, z)=\int_{0}^{\infty} A(\lambda)\left(I_{0}(\lambda \varpi) \cos \lambda z-1\right) d \lambda, \\
& \tau(\varpi, z)=\int_{0}^{\infty} B(\lambda) I_{1}(\lambda \varpi) \cos \lambda z d \lambda,
\end{aligned}
$$

where $I_{0}(\lambda \varpi)$ and $I_{1}(\lambda \varpi)$ are modified Bessel functions of the first kind (7, p. 77). The integrand in the representation for $\chi$ contains $\left(I_{0}(\lambda \varpi) \cos \lambda z-1\right)$ rather than $I_{0}(\lambda \varpi) \cos \lambda z$ to ensure that the integrand does not diverge at the lower limit, it being found later that $A(\lambda)$ is $0\left(\lambda^{-2}\right)$ for small $\lambda$.

The representation (2.3) for $D$ is found to satisfy the required continuity conditions and to tend to zero at a large distance $|z|$ from the origin. Further, at any point of the beam the stresses corresponding to (2.3) are

$$
\begin{aligned}
& \frac{\tilde{z z}}{2 \mu}=\frac{\partial \phi}{\partial z}-z \frac{\partial^{2} \phi}{\partial z^{2}}+\frac{2 \eta}{\varpi} \frac{\partial}{\partial \varpi}(\varpi \tau)-\varpi \frac{\partial^{2} \tau}{\partial z^{2}}+\frac{\partial^{2} \chi}{\partial z^{2}}, \\
& \frac{\tilde{\varpi z}}{2 \mu}=-z \frac{\partial^{2} \phi}{\partial \varpi \hat{\partial} z}+(1-2 \eta) \frac{\partial \tau}{\partial z}-\varpi \frac{\partial^{2} \tau}{\partial \varpi \partial z}+\frac{\partial^{2} \chi}{\partial \varpi \partial z}, \\
& \frac{\tilde{\varpi}}{2 \mu}=2 \eta \frac{\partial \phi}{\partial z}-z \frac{\partial^{2} \phi}{\partial \varpi^{2}}+\frac{\partial^{2} \psi}{\partial \varpi^{2}}+2 \eta \frac{\tau}{\varpi}+2(1-\eta) \frac{\partial \tau}{\partial \varpi}-\varpi \frac{\hat{o}^{2} \tau}{\partial \varpi^{2}}+\frac{\partial^{2} \chi}{\partial \varpi^{2}}, .
\end{aligned}
$$

where $\mu$ is the shear modulus.

\section{The Integral Equation for $g(t)$}

We now show that the representation (2.3) for $D$ satisfies the boundary conditions (2.1) and (2.2) provided $g(t)$ is the solution of a certain Fredholm 
integral equation of the second kind. From (2.8) and (2.10) we have that

$$
\hat{\varpi} z=0 \text { for } z=0(0 \leqq \varpi<\infty),
$$

so that the first of conditions (2.1) is satisfied. Further, from (2.8) and (2.9) the second of these conditions is satisfied provided

$$
\begin{gathered}
\frac{\partial \phi}{\partial z}-\int_{0}^{\infty} \lambda\left[\lambda A(\lambda) I_{0}(\lambda \varpi)-B(\lambda)\left(2 \eta I_{0}(\lambda \varpi)+\lambda \varpi I_{1}(\lambda \varpi)\right)\right] d \lambda=-\frac{p(\varpi)}{2 \mu} \\
\text { for } z=0(0 \leqq \varpi \leqq a) .
\end{gathered}
$$

The first term on the left-hand side of this equation is the limit of $\partial \phi / \partial z$ as the point $(\varpi, \theta, z)$ approaches a point on the crack and is found as

$$
\underset{z \rightarrow 0}{\mathrm{Lt}} \frac{\partial \phi}{\partial z}=\frac{1}{\varpi} \frac{\partial}{\partial \varpi} \int_{0}^{\varpi} \frac{t g(t) d t}{\left(\varpi^{2}-t^{2}\right)^{\frac{1}{2}}} \quad(0 \leqq \varpi \leqq a) .
$$

Thus, on multiplying the above equation by $\varpi$ and integrating with respect to $\varpi$ from 0 to $\varpi$, we obtain

$$
\begin{array}{r}
\int_{0}^{\varpi} \frac{t g(t) d t}{\left(\varpi^{2}-t^{2}\right)^{\frac{1}{2}}}-\varpi \int_{0}^{\infty}\left[\lambda A(\lambda) I_{1}(\lambda \varpi)-B(\lambda)\left(2 \eta I_{1}(\lambda \varpi)+\lambda \varpi I_{2}(\lambda \varpi)\right)\right] d \lambda \\
=-\frac{1}{2 \mu} \int_{0}^{\varpi} \rho p(\rho) d \rho \quad(0 \leqq \varpi \leqq a),
\end{array}
$$

from which we find $g(t)$ in terms of $A(\lambda)$ and $B(\lambda)$ by an application of the known solution of Abel's integral equation (8). We use the integral representations (7, p. 373, equation (1) with $v=-\frac{1}{2}, \mu=\frac{1}{2}$ and $\frac{3}{2}$ )

$$
\begin{aligned}
\varpi I_{1}(\lambda \varpi) & =\frac{2}{\pi} \int_{0}^{\varpi} \frac{t \sinh \lambda t}{\left(\varpi^{2}-t^{2}\right)^{\frac{1}{2}}} d t, \\
\varpi^{2} I_{2}(\lambda \varpi) & =\frac{2}{\pi} \int_{0}^{\varpi} \frac{t^{2}}{\left(\varpi^{2}-t^{2}\right)^{\frac{1}{2}}}\left(\cosh \lambda t-\frac{\sinh \lambda t}{\lambda t}\right) d t,
\end{aligned}
$$

to obtain

$g(t)-\frac{2}{\pi} \int_{0}^{\infty}[\lambda A(\lambda) \sinh \lambda t+B(\lambda)((1-2 \eta) \sinh \lambda t-\lambda t \cosh \lambda t)] d \lambda=P(t)$

$(0 \leqq t \leqq a)$,

where

$$
P(t)=-\frac{1}{\pi \mu} \int_{0}^{t} \frac{\varpi p(\varpi) d \varpi}{\left(t^{2}-\varpi^{2}\right)^{\frac{1}{2}}} \quad(0 \leqq t \leqq a) .
$$

We now obtain two further equations relating the three functions $g(t)$, $A(\lambda)$ and $B(\lambda)$ from the condition that the curved surface of the beam is 
stress-free. From $(2.10) \hat{\varpi z}$ is zero on $\varpi=c(-\infty<z<\infty)$ provided

$$
\begin{aligned}
\int_{0}^{\infty}\left[\lambda A(\lambda) I_{1}(\lambda c)-B(\lambda)\left(\lambda c I_{0}(\lambda c)-\right.\right. & \left.\left.2(1-\eta) I_{1}(\lambda c)\right)\right] \lambda \sin \lambda z d \lambda \\
& =-\frac{3 c z}{2 i} \int_{-a}^{a} \frac{g(t)(z+i t) d t}{\left(c^{2}+(z+i t)^{2}\right)^{\frac{3}{2}}} \quad(-\infty<z<\infty) .
\end{aligned}
$$

An application of the Fourier sine theorem gives

$$
\begin{aligned}
& \lambda^{2} A(\lambda) I_{1}(\lambda c)-\lambda B(\lambda)\left(\lambda c I_{0}(\lambda c)-2(1-\eta) I_{1}(\lambda c)\right) \\
& =\frac{1}{i \pi} \int_{0}^{a} g(s) S(\lambda, s) d s, \quad(0 \leqq \lambda<\infty)
\end{aligned}
$$

where

$$
S(\lambda, s)=\int_{0}^{\infty}\left(\frac{3 c z(z-i s)}{\left(c^{2}+(z-i s)^{2}\right)^{\frac{3}{2}}}-\frac{3 c z(z+i s)}{\left(c^{2}+\left(z+i s^{2}\right)^{\frac{3}{2}}\right)}\right) \sin \lambda z d z .
$$

We evaluate this integral, making use of the real and imaginary parts of the integrals (7, p. 410, equation (2) with $v=\frac{1}{2}, \mu=1$ and 2)

$$
\begin{aligned}
& \frac{2}{\pi} \int_{0}^{\infty} \lambda K_{1}(\lambda c) \cos \lambda u d \lambda=\frac{c}{\left(c^{2}+u^{2}\right)^{\frac{1}{2}}}, \\
& \frac{2}{\pi} \int_{0}^{\infty} \lambda^{2} K_{2}(\lambda c) \cos \lambda u d \lambda=\frac{3 c^{2}}{\left(c^{2}+u^{2}\right)^{\frac{3}{2}}}
\end{aligned}
$$

where $c>|\operatorname{Im} u|$ and $K_{1}(\lambda c)$ and $K_{2}(\lambda c)$ are modified Bessel functions of the third kind $(7$, p. 78$)$, to find

$$
S(\lambda, s)=2 i \lambda\left[\left(3 K_{1}(\lambda c)-\lambda c K_{2}(\lambda c)\right) \sinh \lambda s+K_{1}(\lambda c) \lambda s \cosh \lambda s\right],
$$

provided $c>s$, a condition which is satisfied since $c>a \geqq s$. Hence equation (3.4) becomes

$$
\begin{aligned}
\lambda A(\lambda) I_{1}(\lambda c)- & B(\lambda)\left(\lambda c I_{0}(\lambda c)-2(1-\eta) I_{1}(\lambda c)\right)=\frac{2}{\pi} K_{1}(\lambda c) \int_{0}^{a} g(s) \lambda s \cosh \lambda s d s \\
+ & \frac{2}{\pi}\left(3 K_{1}(\lambda c)-\lambda c K_{2}(\lambda c)\right) \int_{0}^{a} g(s) \sinh \lambda s d s \quad(0 \leqq \lambda<\infty) . \ldots
\end{aligned}
$$

Finally, from (2.11) $\tilde{\varpi \varpi}$ is zero on $\varpi=c(-\infty<z<\infty)$ provided

$$
\begin{array}{r}
\int_{0}^{\infty}\left[\lambda A(\lambda)\left(\lambda c I_{0}(\lambda c)-I_{1}(\lambda c)\right)+B(\lambda)\left((3-2 \eta) \lambda c I_{0}(\lambda c)-4(1-\eta) I_{1}(\lambda c)\right.\right. \\
\left.\left.-(\lambda c)^{2} I_{1}(\lambda c)\right)\right] \cos \lambda z d \lambda \\
=\frac{c}{2 i} \int_{-a}^{a} g(t)\left[\frac{(1-2 \eta)(z+i t)}{c^{2}\left(c^{2}+(z+i t)^{2}\right)^{\frac{1}{2}}}+\frac{i t}{\left(c^{2}+(z+i t)^{2}\right)^{\frac{2}{2}}}+\frac{3 c^{2} z}{\left(c^{2}+(z+i t)^{2}\right)^{\frac{3}{2}}}\right] d t .
\end{array}
$$


W. D. COLLINS

We now apply the Fourier cosine theorem and make use of the integrals (3.5) and (3.6) to obtain

$$
\begin{aligned}
\lambda A(\lambda)\left(\lambda c I_{0}(\lambda c)-I_{1}(\lambda c)\right)+B(\lambda)\left((3-2 \eta) \lambda c I_{0}(\lambda c)-4(1-\eta) I_{1}(\lambda c)-(\lambda c)^{2} I_{1}(\lambda c)\right) \\
\quad=\frac{2}{\pi}\left(K_{1}(\lambda c)-\lambda c K_{2}(\lambda c)\right) \int_{0}^{a} g(s) \lambda s \cosh \lambda s d s \\
+\frac{2}{\pi}\left(1-2 \eta+(\lambda c)^{2}\right) K_{1}(\lambda c) \int_{0}^{a} g(s) \sinh \lambda s d s \quad(0 \leqq \lambda<\infty) . \ldots \ldots . . .
\end{aligned}
$$

We solve the simultaneous equations (3.7) and (3.8) for $A(\lambda)$ and $B(\lambda)$ to find

$$
\begin{aligned}
& \lambda A(\lambda) G(\lambda c)=\frac{2}{\pi}(2-2 \eta-H(\lambda c)) \int_{0}^{a} g(s) \lambda s \cosh \lambda s d s \\
& +\frac{2}{\pi}\left(4-4 \eta+(\lambda c)^{2}-(3-2 \eta) H(\lambda c)\right) \int_{0}^{a} g(s) \sinh \lambda s d s,
\end{aligned}
$$

where

$$
\begin{array}{r}
G(\lambda c)=(\lambda c)^{2} I_{0}^{2}(\lambda c)-\left(2-2 \eta+(\lambda c)^{2}\right) I_{1}^{2}(\lambda c), \ldots \ldots \ldots \\
H(\lambda c)=(\lambda c)^{2} I_{0}(\lambda c) K_{0}(\lambda c)+\left(2-2 \eta+(\lambda c)^{2}\right) I_{1}(\lambda c) K_{1}(\lambda c) .
\end{array}
$$

For small values of $\lambda A(\lambda)$ and $B(\lambda)$ are $O\left(\lambda^{-2}\right)$ and $O\left(\lambda^{-1}\right)$ respectively. The integrands of $\chi$ and $\tau$ given by (2.8) are however finite at $\lambda=0$, so that the integrals for these functions do not diverge at the lower limits.

On substituting the expressions (3.9) and (3.10) into (3.2) we obtain a Fredholm integral equation of the second kind determing $g(t)$, this being

where

$$
g(t)+\frac{2}{\pi} \int_{0}^{a} K(t, s) g(s) d s=P(t) \quad(0 \leqq t \leqq a),
$$

$$
\begin{aligned}
& K(t, s)=\frac{2}{\pi} \int_{0}^{\infty}[G(\lambda c)]^{-1}\left[\left(2 H(\lambda c)-3+2 \eta-(\lambda c)^{2}\right) \sinh \lambda t \sinh \lambda s\right. \\
& \left.\quad-\lambda^{2} t s \cosh \lambda t \cosh \lambda s+(H(\lambda c)-1)(\lambda t \cosh \lambda t \sinh \lambda s+\lambda s \cosh \lambda s \sinh \lambda t)\right] d \lambda .
\end{aligned}
$$

Once $g(t)$ is found, $A(\lambda)$ and $B(\lambda)$ follow from (3.9) and (3.10) and the stresses and displacement at any point can then be calculated. The exact solution of (3.13) is not however known, and it is necessary to resort to numerical and approximate methods to solve it. In particular, when $c$ tends to infinity, $K(t, s)$ tends to zero and (3.13) reduces to the corresponding equation for an infinite solid containing a crack opened under normal pressure obtained previously (1, equation (2.12)). Thus, when the ratio $a / c$ is sufficiently small, an iterative solution of (3.13), perturbing on the solution for a crack in an 
infinite solid, can be obtained as a convergent power series, the first few terms of which provide a good approximation to $g(t)$.

To derive this solution we first expand $K(t, s)$ in powers of $c^{-1}$. We find that

$$
\begin{aligned}
K(t, s)=\frac{2}{\pi c} \int_{0}^{\infty} & G^{-1}(\xi)\left[\left(2 H(\xi)-3+2 \eta-\xi^{2}\right) \sinh \left(\frac{\xi t}{c}\right) \sinh \left(\frac{\xi s}{c}\right)\right. \\
& -\frac{\xi^{2} t s}{c^{2}} \cosh \left(\frac{\xi t}{c}\right) \cosh \left(\frac{\xi s}{c}\right)+(H(\xi)-1)\left(\frac{\xi t}{c} \cosh \left(\frac{\xi t}{c}\right) \sinh \left(\frac{\xi s}{c}\right)\right. \\
& \left.\left.+\frac{\xi s}{c} \cosh \left(\frac{\xi s}{c}\right) \sinh \left(\frac{\xi t}{c}\right)\right)\right] d \xi \\
& \left.=\sum_{r=0}^{\infty} c^{-2 r-3} \sum_{n=0}^{r} D_{r-n, n} t^{2 n+1} s^{2 r-2 n+1}, \ldots \ldots \ldots \ldots \ldots \ldots . .13 .15\right)
\end{aligned}
$$

where

$$
\begin{array}{r}
D_{m n}=D_{n m}=2\left[(m+n+2) E_{m n}-[2(m+1)(n+1)+1-\eta] F_{m n}\right. \\
\left.-(m+1)(2 m+3) F_{m+1, n}\right],
\end{array}
$$

with

and

$$
E_{m n}=E_{n m}=\frac{2}{\pi} \frac{1}{(2 m+1) !(2 n+1) !} \int_{0}^{\infty} \frac{\xi^{2(m+n+1)} H(\xi)}{G(\xi)} d \xi
$$

$$
F_{m n}=F_{n m}=\frac{2}{\pi} \frac{1}{(2 m+1) !(2 n+1) !} \int_{0}^{\infty} \frac{\xi^{2(m+n+1)}}{G(\xi)} d \xi .
$$

For a given value of $\eta$ the coefficients $E_{m n}$ and $F_{m n}$ need to be evaluated numerically for various $m$ and $n$ depending on the order of the terms in (3.15) neglected in obtaining an approximate solution of (3.13). We can however express $E_{m n}$ and $F_{m n}$ in terms of some related coefficients ${ }^{2 m} \alpha_{2 n}$ and ${ }^{2 m} \gamma_{2 n}$, which arise in a paper by Ling (5, equation (21)) on a spherical cavity in a circular beam under tension and then make use of Ling's numerical results for these coefficients to calculate $E_{m n}$ and $F_{m n}$ and hence $D_{m n}$. In this way we find for instance that, when $\eta=0 \cdot 3$,

$$
D_{00}=-2 \cdot 4751, D_{10}=-0.3938 \text {. }
$$

\section{The Crack Opened under Constant Pressure}

When the crack is opened by a constant pressure $p$, we have

so that from (3.3)

$$
p(w)=p,
$$

$$
P(t)=-\frac{p t}{\pi \mu}
$$

and the Fredholm equation (3.13) determining $g(t)$ is

$$
g(t)+\frac{2}{\pi} \int_{0}^{a} K(t, s) g(s) d s=-\frac{p t}{\pi \mu} \quad(0 \leqq t \leqq a) .
$$


The approximate solution of this equation obtained by iteration is

$$
\begin{aligned}
g(t)= & -\frac{p t}{\pi \mu}\left[1-\frac{2 D_{00} a^{3}}{3 \pi c^{3}}-\frac{2 D_{10} a^{3}}{3 \pi c^{5}}\left(t^{2}+\frac{3 a^{2}}{5}\right)+\frac{4 D_{00}^{2} a^{6}}{9 \pi^{2} c^{6}}\right. \\
& -\frac{2 a^{3}}{\pi c^{7}}\left(\frac{D_{20} t^{4}}{3}+\frac{D_{11} t^{2} a^{2}}{5}+\frac{D_{20} a^{4}}{7}\right)+\frac{4 D_{00} D_{10} a^{6}}{9 \pi^{2} c^{8}}\left(t^{2}+\frac{9 a^{2}}{5}\right) \\
& -\frac{2 a^{3}}{\pi c^{9}}\left(\frac{D_{30} t^{6}}{3}+\frac{D_{21} t^{4} a^{2}}{5}+\frac{D_{21} t^{2} a^{4}}{7}+\left(D_{30}+\frac{4 D_{00}^{3}}{3 \pi^{2}}\right) \frac{a^{6}}{9}\right) \\
& +\frac{4 a^{6}}{\pi^{2} c^{10}}\left(\frac{D_{00} D_{20} t^{4}}{9}+\left(D_{00} D_{11}+2 D_{10}^{2}\right) \frac{t^{2} a^{2}}{15}\right. \\
& \left.\left.+\left(\frac{D_{00} D_{20}}{7}+\frac{46 D_{10}^{2}}{525}+\frac{D_{00} D_{11}}{25}\right) a^{4}\right)\right]+O\left(c^{-11}\right) .
\end{aligned}
$$

From (2.3), (2.4) and (2.6) we find the $z$-component $w$ of the displacement as

$$
w=0 \text { for } z=0(m>a),
$$

and

$$
w_{ \pm}=\mp 2(1-\eta) \int_{w}^{a} \frac{g(t) d t}{\left(t^{2}-w^{2}\right)^{\frac{1}{2}}} \text { for } z=0_{ \pm}(0 \leqq \varpi \leqq a),
$$

the upper and lower signs holding for points on the faces $z=0_{+}$and $z=0_{-}$ of the crack respectively. Hence, using (4.2), we find that

$$
\begin{aligned}
& w_{ \pm}= \pm \frac{2 p(1-\eta)\left(a^{2}-\varpi^{2}\right)^{\frac{1}{2}}}{\pi \mu}\left[1-\frac{2 D_{00} a^{3}}{3 \pi c^{3}}-\frac{4 D_{10} a^{3}}{9 \pi c^{5}}\left(\varpi^{2}+\frac{7 a^{2}}{5}\right)+\frac{4 D_{00}^{2} a^{6}}{9 \pi^{2} c^{6}}\right. \\
& -\frac{2 a^{3}}{15 \pi c^{7}}\left(\frac{8 D_{20}}{3} \pi^{4}+\left(\frac{4}{3} D_{20}+2 D_{11}\right) \varpi^{2} a^{2}+\left(\frac{22 D_{20}}{7}+D_{11}\right) a^{4}\right) \\
& +\frac{8 D_{00} D_{10} a^{6}}{27 \pi^{2} c^{8}}\left(\varpi^{2}+\frac{16 a^{2}}{5}\right)-\frac{2 a^{3}}{\pi c^{9}}\left(\frac{16 D_{30} \varpi^{6}}{105}+\left(\frac{8 D_{30}}{105}+\frac{8 D_{21}}{75}\right) \varpi^{4} a^{2}\right. \\
& \left.+\left(\frac{2 D_{30}}{35}+\frac{26 D_{21}}{175}\right) \varpi^{2} a^{4}+\left(\frac{10 D_{30}}{63}+\frac{46 D_{21}}{525}+\frac{4 D_{00}^{3}}{27 \pi^{2}}\right) a^{6}\right) \\
& +\frac{8 a^{6}}{15 \pi^{2} c^{10}}\left(\frac{4 D_{00} D_{20}}{9} \varpi^{4}+\left(\frac{2}{9} D_{00} D_{20}+\frac{2}{3} D_{10}^{2}+\frac{1}{3} D_{00} D_{11}\right) \varpi^{2} a^{2}\right. \\
& \left.\left.+\left(\frac{26}{21} D_{00} D_{20}+\frac{104}{105} D_{10}^{2}+\frac{7}{15} D_{00} D_{11}\right) a^{4}\right)\right]+O\left(c^{-11}\right) \text {. }
\end{aligned}
$$

Values of $w_{+}$for varous $\boldsymbol{w}$ when $c=5 a$ and $\eta=0.3$ are given in Table $I$ and are found to be about 0.5 per cent. greater than the corresponding values for the normal displacement over a crack in an infinite solid given by (4.4) when $c$ tends to infinity. 
TABLE I

Values of $w_{+} / D$ for various $\varpi$ when $a / c=0,0 \cdot 2$, and $\eta=0 \cdot 3$.

$$
\pi \mu D=2(1-\eta) p a
$$

\begin{tabular}{|c|c|c|}
\hline$\varpi / a$ & $a / c=0$ & $a / c=0.2$ \\
\hline 0.0 & 1.0000 & 1.0042 \\
0.1 & 0.9950 & 0.9991 \\
0.2 & 0.9798 & 0.9839 \\
0.3 & 0.9539 & 0.9579 \\
0.4 & 0.9165 & 0.9203 \\
0.5 & 0.8660 & 0.8696 \\
0.6 & 0.8000 & 0.8033 \\
0.7 & 0.7141 & 0.7171 \\
0.8 & 0.6000 & 0.6025 \\
0.9 & 0.4359 & 0.4377 \\
1.0 & 0 & 0 \\
\hline
\end{tabular}

The increase $W$ in the potential energy of deformation due to the crack is (3, p. 173)

$$
\begin{aligned}
& W=\pi p \int_{0}^{a} m\left(w_{+}-w_{-}\right) d \varpi \\
& =-4 \pi p(1-\eta) \int_{0}^{a} t g(t) d t \\
& =\frac{4(1-\eta) p^{2} a^{3}}{3 \mu}\left[1-\frac{2 D_{00}}{3 \pi} \sigma^{3}-\frac{4 D_{10}}{5 \pi} \sigma^{5}+\frac{4 D_{00}^{2}}{9 \pi^{2}} \sigma^{6}-\frac{2}{\pi}\left(\frac{2 D_{20}}{7}+\frac{3 D_{11}}{25}\right) \sigma^{7}\right. \\
& +\frac{16 D_{00} D_{10}}{15 \pi^{2}} \sigma^{8}-\frac{4}{\pi}\left(\frac{1}{9} D_{30}+\frac{3}{35} D_{21}+\frac{2}{27 \pi^{2}} D_{00}^{3}\right) \sigma^{9} \\
& \left.+\frac{8}{\pi^{2}}\left(\frac{2 D_{00} D_{20}}{21}+\frac{D_{00} D_{11}}{25}+\frac{44 D_{10}^{2}}{525}\right) \sigma^{10}+O\left(\sigma^{11}\right)\right]
\end{aligned}
$$

where $\sigma=a / c$. When $\sigma=0 \cdot 2$ and $\eta=0 \cdot 3$, we have

$$
\mu W=0.9373 p^{2} a^{3} \text {. }
$$

\section{A Crack in a Beam under Tension}

The effect of a crack on a circular beam under a uniform tension in the $z$-direction can be deduced immediately from the previous analysis. If the solution

$$
\hat{z z}=p, \hat{\varpi z}=\hat{\varpi \varpi}=0,
$$

is added to the stresses given by (2.9), (2.10), and (2.11) for the crack opened 
under constant pressure $p$, the resuiting stress distribution is that for a crack in a circular beam under a uniform tension $p$ in the $z$-direction, the surfaces of the crack and the beam being stress-free. The presence of the crack lowers the potential energy of the beam by an amount $W$ given by (4.5). The criterion given by Griffith (9) that the crack may spread is

$$
\frac{\partial}{\partial a}(W-U)=0 \text {, }
$$

where the surface energy $U$ of the crack is given by

$$
U=2 \pi a^{2} T \text {, }
$$

$T$ being the surface tension of the elastic material. Thus for small values of the ratio $\sigma=a / c$ the crack will become unstable and spread if $p$ exceeds the critical value

$$
\begin{array}{r}
p_{c r}=p_{0}\left[1+\frac{2 D_{00}}{3 \pi} \sigma^{3}+\frac{16 D_{10}}{15 \pi} \sigma^{5}+\frac{2}{\pi}\left(\frac{10}{21} D_{20}+\frac{1}{5} D_{11}\right) \sigma^{7}+\frac{8 D_{00} D_{10}}{45 \pi^{2}} \sigma^{8}\right. \\
+\frac{8}{\pi}\left(\frac{1}{9} D_{30}+\frac{3}{35} D_{21}\right) \sigma^{9}+\frac{8}{3 \pi^{2}}\left(\frac{2}{21} D_{00} D_{21}+\frac{1}{25} D_{00} D_{11}+\frac{2}{21} D_{10}^{2}\right) \sigma^{10} \\
\left.+O\left(\sigma^{11}\right)\right]
\end{array}
$$

where

$$
p_{0}=\left(\frac{\pi \mu T}{(1-\eta) a}\right)^{\frac{1}{2}}
$$

is the corresponding critical value of $p$ for a crack in an infinite solid under a uniform tension (9).

When $\eta=0 \cdot 3,(3.19)$ shows that $D_{00}$ is negative and hence $p_{c r}$ is less than $p_{0}$. In particular, when $\sigma=0 \cdot 2$,

$$
p_{c r}=0.9958 p_{0} \text {. }
$$

\section{REFERENCES}

(1) W. D. Collins, Proc. Royal Soc. A 266 (1962), 359-386.

(2) W. D. Colurns, Mathematika (to be published).

(3) A. E. H. Love, Mathematical Theory of Elasticity, 4th edn. (Cambridge, 1952).

(4) C. J. Tranter and J. W. Craggs, Phil. Mag. (7), 36 (1945), 241-250.

(5) C.-B. Ling, Q. App. Maths., 13 (1956), 381-391.

(6) M. V. BARTON, J. App. Mechs., 8 (1941), 97-104.

(7) G. N. Warson, Theory of Bessel Functions, 2nd edn. (Cambridge, 1958).

(8) E. T. Copson, Proc. Edin. Math. Soc. (2), 8 (1947-50), 14-19.

(9) I. N. SNeddon, Proc. Royal Soc. A 187 (1946), 229-260.

THE UNIVERSITY

MANCHESTER 